\title{
Preventing mussel loss
}

The Great Lakes are an area of high conservation concern as their collective ecosystem now hosts more than 180 invasive and nonnative species that continue to transform food webs and decimate native species. Two infamous examples of such invaders are the zebra mussels (Dreissena polymorpha) and quagga mussels (Dreissena bugensis) that became established in the late 1990s and have substantially altered the habitat and ecosystem of the Great Lakes.

Many of the victims of this particular event were the lakes' native unionid mussel species, which were quickly extirpated from the lakes and now persist, imperiled, in minor refugia offered by tributaries and bays. Unionid mussels are particularly dependent upon their native ecological communities, as their larvae, called glochidia, are obligate parasites that depend on vertebrate hosts in order to grow and metamorphose into juvenile mussels.

The round goby (Neogobius melanostomus) was also introduced to the Great Lakes in the 1990s and is now expanding into the

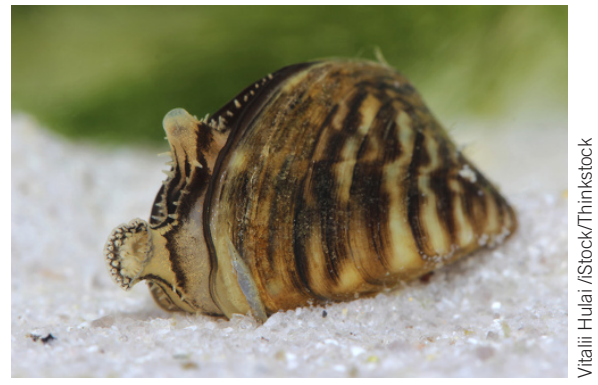

carry out laboratory infections of 3 types of fish for each species: a native primary host of the species' larvae, a native marginal host and the invasive round goby ( $R$. Soc. Open Sci. 3, 150481; 2016). The researchers measured the infestation rate and metamorphosis rate of the glochidia and, finally, number of juveniles that were produced per fish.

These data, alongside field experiments, indicated that many species of unionid mussels infest round gobies at rates that are comparable to those at which they infest their primary or marginal hosts; however, very few of these glochidia successfully metamorphose to the juvenile life stage. This suggests that round gobies act as a sink for these threatened mussels, and that their presence in refugia habitats will attract unionid larvae without contributing to the juvenile population. Processes such as this, when seen under laboratory conditions, can allow biologists and watershed management teams to better predict, understand and hopefully mitigate the fallout of unfolding biological invasions.

Gregory D. Larsen

\section{MUTANT MOUSE MODELSTUTTERS IN SOUUEAKS}

Stuttering is a speech disorder in which the flow of speech is disrupted by frequent repetitions, prolongations or hesitations. Scientists do not completely understand the precise reasons why some people experience stuttering, but recent research has found that among cases of persistent stuttering in the absence of other symptoms, 9-16\% of cases are associated with mutations in the lysosomal enzyme-targeting pathway. This pathway is responsible for cellular 'housekeeping' and depends on the products of three particular genes; among humans who stutter, mutations have been found in all three of these genes.

Terra Barnes and peers at Washington University (St. Louis, MO) and the National Institutes of Health (Bethesda, MD) recreated this genetic condition in mice by creating a knock-in mutation in the gene Gnptab (Curr. Biol. doi:10.1016/j.cub.2016.02.068; published online 25 April 2016). This gene, when mutated, is known to cause stuttering in human speech, and the researchers hoped that a similar disruption would occur in the vocalizations of the mutant mice. To test this, they isolated pups from their mothers in order to elicit and record vocalizations known as 'isolation calls'.

These mutant mice did, indeed, produce distinctive vocalizations that, according to Barnes, "mimic some features of human stuttering." These mice had significantly fewer vocalizations with a higher incidence of long pauses, compared to wild-type littermates. Mutants also showed signs of temporal stereotypy in their bouts of vocalizations, repeating the same syllables more often and with less diversity than their wild-type peers.

Like stuttering humans, these mice showed no obvious non-vocal abnormalities, which suggests that this model might reproduce non-syndromic stuttering as it occurs in humans, through similar changes in the lysosomal enzyme-targeting pathway. In a press release, senior author Timothy Holy suggested how this might come about: "It could be that the protein has many functions and this mutation affects only one of them. Or the mutation could very mildly compromise the function of the protein, but there's a set of cells in the brain that is exquisitely sensitive, and if you ever so slightly compromise the function in those cells you get the observable behavioral deficit."

If this murine phenotype does in fact model stuttering in humans, it could help to establish a neurophysiological basis for this condition, and Barnes, Holy and their team are already planning next steps to tease apart potential causes and mechanisms of this very specific communicative disorder. 